\title{
What Is the Appropriate Acupuncture Treatment Schedule for Chronic Pain? Review and Analysis of Randomized Controlled Trials
}

\author{
Yan-Jiao Chen, Cai-Tao Chen, Jia-Yuan Liu $\mathbb{D}$, \\ Gabriel Shimizu Bassi, and Yong-Qing Yang $\mathbb{D}$ \\ Shanghai Research Institute of Acupuncture and Meridian, Shanghai University of Traditional Chinese Medicine, \\ 650 South Wanping Road, Shanghai 200030, China \\ Correspondence should be addressed to Yong-Qing Yang; yyq@shutcm.edu.cn
}

Received 1 February 2019; Revised 20 May 2019; Accepted 29 May 2019; Published 18 June 2019

Academic Editor: Arthur De Sá Ferreira

Copyright (C) 2019 Yan-Jiao Chen et al. This is an open access article distributed under the Creative Commons Attribution License, which permits unrestricted use, distribution, and reproduction in any medium, provided the original work is properly cited.

\begin{abstract}
Background. Acupuncture is widely used for the treatment of chronic pain. Different protocols of acupuncture practice exist and lack agreement on the optimal schedule of acupuncture treatment. Objective. To review the appropriate acupuncture treatment schedule for chronic pain. Methods. Embase, Pubmed, Cochrane Central Register of Controlled Trials, and reference lists were searched from 2009 to 2018 to identify randomized controlled trials of acupuncture for chronic pain conditions. We collected factors of treatment schedule ( $D$, duration of each treatment session; $N$, number of treatment sessions; $T$, total duration of treatment in weeks) from each of the trials, and the linear regression analysis with real pain relief rate (both treatment and follow-up) was performed. Furthermore, we recommend the concept of "DOSE" and frequency (F) to evaluate the dose and frequency effect of acupuncture. Results. Twentyfour trials with a total number of 3461 patients met the inclusion criteria. Of these, data from 23 studies were available for analysis. Firstly, the results showed that follow-up pain relief rate was decreased slightly with the increase of the duration of each session and DOSE ( $r=-0.3414$ and $r=-0.3246$, respectively), but those two factors had no correlation with the pain relief rate after treatment. Secondly, it showed that either lower frequency with 2 sessions/week and higher frequency greater than 2 sessions/week or DOSE of $30 \mathrm{mins} /$ week can achieve higher pain relief rate after treatment. Thirdly, we found the rate of pain relief remained at a high level greater than $20 \%$ up to 18 weeks after the treatment, and then it dropped sharply below $10 \%$ with the follow-up extended. A positive relationship was found between study score and pain relief both in treatment and follow-up ( $\mathrm{r}=0.4654$ and $\mathrm{r}=0.3046$, respectively). Conclusions. The effect of acupuncture varies greatly with the different schedules of acupuncture, so it is necessary to review and choose the appropriate schedule. Although the current work is based on a limited number of trials, the findings suggest that acupuncture has a dose and frequency effect presenting within a certain range, which would have considerable implications for the design and interpretation of clinical trials. More high-quality randomized controlled trials on acupuncture schedule research were needed for providing more definitive evidence.
\end{abstract}

\section{Introduction}

Acupuncture has been used for more than 2500 years in China [1]. The World Health Organization (WHO) listed pain and other 42 indications for acupuncture in 1979 [2] and 107 diseases or disorders in 2002 [3]. A recent study found pain represents the largest category among the top 99 indications in private clinics in the United States [4]. Meanwhile, the National Academies of Sciences, Engineering, and Medicine (NASEM) pointed out that acupuncture has become common practice for pain relief in recent decades [5]. Acupuncture is now widely used as a powerful tool for controlling pain conditions.

In many of the pain conditions where acupuncture is used, there is limited high-quality evidence to draw clear conclusions over its effectiveness $[6,7]$. Researchers argue that the efficacy of acupuncture is difficult to be confirmed in clinical trials because a reasonable effective control group was used [8]. More researchers are studying how sham acupuncture, the most commonly used control in acupuncture research, should be operated to avoid producing specific effects of acupuncture resulting from blunt needles [9], 
needled outside known points [10], and acupoints not indicated for conditions [11]. However, those sham acupuncture methods should not be used by acupuncture proponents to explain negative results unless when they are supported by evidence.

Other than the control methods, there are many other factors affecting the efficacy of acupuncture, one of which is that the treatment protocol cannot be unified. Different clinical trials used different protocols and a protocol used by an acupuncturist might be dismissed by another $[12,13]$. In 2010, the revised STandards for Reporting Interventions in Clinical Trials of Acupuncture (STRICTA) were published to encourage the publication protocol of precise interventions methods used in trials of acupuncture [14]. The recommendations emphasized the need to provide rationed detailed protocol information, such as needling, treatment regimen, cointerventions, practitioners background, and control interventions. However, suggested schedule, the key component of protocol, including the range of duration of each treatment session, number of treatment sessions, and total duration of treatment in weeks for diseases, is not given.

The effect of factors of schedule on acupuncture was ignored for a long time. In the present study, a review of acupuncture randomized controlled trials for the treatment of chronic pain conditions was conducted from 2009 to 2018. In addition, schedule related factors influencing pain relief during both acupuncture treatment and follow-up period were investigated.

\section{Methods}

2.1. Selection and Exclusion Criteria. To be included, studies met the following criteria: (1) randomized controlled trials; (2) pain-related problems (for example, knee osteoarthritis, low back pain, fibromyalgia, shoulder pain, subacromial impingement, neck pain, myofascial pain, pelvic pain, headache, and plantar fasciitis); (3) patients $\geq 18$ years old; (4) classical acupuncture intervention (only needles); (5) chronic pain (pain lasting more than 3 months).

Excluding criteria are as follows: (1) healthy volunteers and pregnancy; (2) cancer and menstrual pain; (3) auricular, dry needling, tongue, microsystems, intradermal, laser, acupressure, apipuncture, scalp, facial, and electrical acupuncture.

2.1.1. Type of Controls. Inclusion criteria are as follows: (1) no treatment or waiting list; (2) usual care (including medicine therapy); (3) physiotherapy; (4) relaxation; (5) self-educational programs; (6) manipulation; (7) superficial acupuncture; (8) nonpenetrating needles; (9) insertion simulation at nonacupoints/acupoints away; (10) application of placebo TENS or laser; (11) exercise.

Exclusion criteria are as follows: (1) no control; (2) same acupuncture with different number of needles/sessions.

2.2. Data Sources and Searches. We searched Embase database, Pubmed database, and the Cochrane Central
Register of Controlled Trials from 2009 to 2018. Our search strategy was selected by the iteration of the words "chronic pain", "acupuncture", etc. (detailed retrieval type was provided in Supplementary File 1). Chinese trials and trials from Chinese databases were not considered. Trials were firstly selected by screening the titles and abstracts of all references and assessed by two reviewers. If included in the study, the article is fully checked. Any disagreement was solved by discussion and reanalysis of the data.

2.3. Data Extraction and Quality Assessment. The following aspects were considered: $\mathrm{N}$, number of treatment sessions; $\mathrm{D}$, duration of each treatment session; $\mathrm{T}$, total duration of treatment in weeks; results on the pain outcome measures; follow-up pain outcomes; and supplement data. A second reviewer checked all extracted trials results against the original publications.

For the pain evaluation scale, we used the following priority order: Visual Analogue Scale (VAS), Western Ontario and McMaster Universities Osteoarthritis (WOMAC), Northwick Park Questionnaire (NPQ), Short Form Health Survey (SF36), Shoulder Pain and Disability Index (SPADI), National Institutes of Health Chronic Prostatitis Symptom Index (NIH-CPSI), Symptom Bothersomeness Scale, and Knee Society Score (KSS). In addition, the following strategy for pain outcome was used: (1) If the pain-related scale was a comprehensive scale, the part about the pain measurement was selected (for example, in WOMAC, we chose the part of mean WOMAC pain). (2) Both treatment pain relief score and follow-up pain relief score should be as close as possible to the completion of the treatment and follow-up. (3) Missing data were acquired by contacting the original study authors. If the missing data cannot be obtained, we analyzed the available data (disagreements were solved by discussion). (4) When meeting the interval value parameters, mean values were used.

2.3.1. Quality Assessment. Two reviewers independently evaluated the methodological quality of the included studies using the combination of Cochrane risk of bias tool [15] and the completeness of the STandards for Reporting Interventions in Clinical Trials of Acupuncture (STRICTA) checklist. Any disagreement was solved by discussion and reanalysis of the data.

2.4. Data Synthesis and Analysis. Statistical significance of differences between the results was tested using the oneway ANOVA. Linear regression was used to model the relationship between two variables. Results were considered significant when $\mathrm{P}<0.1$. This review is reported in accord with Preferred Reporting Items for Systematic Reviews and Meta-Analyses (PRISMA).

2.4.1. Measures of Treatment Effect. (1) Pain relief rate was calculated based on the following formula.

$$
\text { Pain relief rate }=\left|\frac{\text { Mean Pain }(\text { treatment } / \text { follow-up })-\text { Mean Pain (baseline })}{\text { Mean Pain (baseline) }} \times 100 \%\right|
$$




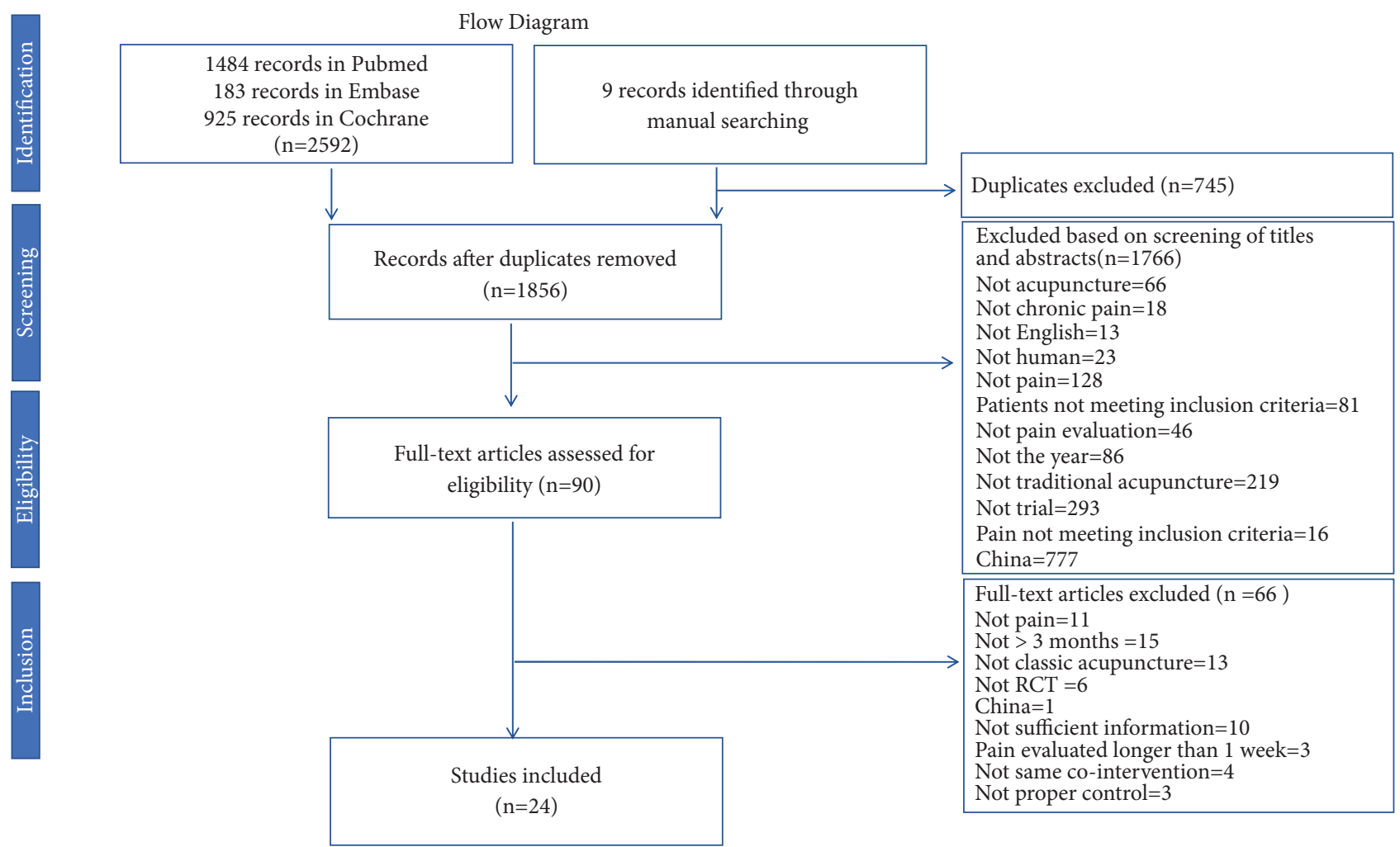

FIGURE 1: Flow diagram of trials selection process.

(2) Real pain relief rate was calculated based on the following formula.

$$
\begin{aligned}
& \text { Real pain relief rate } \\
& =\text { Pain relief rate }{ }^{\text {acupuncture }}-\text { Pain relief rate }{ }^{\text {control }}
\end{aligned}
$$

2.4.2. Assessment of Treatment Schedule. (1) The concept of "DOSE" for measuring the dose of acupuncture was defined, which was calculated based on the following formula: DOSE $=\mathrm{D} \times \mathrm{N} / \mathrm{T}(\mathrm{D}$, duration of each treatment session; $\mathrm{N}$, number of treatment sessions; $\mathrm{T}$, total duration of treatment in weeks).

(2) We used a similar concept for calculating the frequency of treatment based on the following formula: $\mathrm{F}=$ $\mathrm{N} / \mathrm{T}$ (F, frequency; $\mathrm{N}$, number of treatment sessions; $\mathrm{T}$, total duration of treatment in weeks).

\section{Results}

3.1. Literature Search and Description of Included Studies. Literature search identified 2592 references by using search method and 9 others were further added by manual search. 745 duplicates were excluded and 1766 studies were removed by screening. 90 studies were assessed and further analysis excluded 66 records; a total of 24 articles were included finally. The process of study selection was described in Figure 1.

In twenty-four trials, a total of 3461 patients (median 64, minimum 16, and maximum 638) were assessed (Table 1).
Four trials addressed headache (737 patients) and fibromyalgia (319 patients); three trials addressed low back pain (807 patients), headache (237 patients), and knee osteoarthritis (551 patients); two addressed neck pain (556 patients) and jaw pain (44 patients); and one addressed arm pain (50 patients), myofascial pain (60 patients), and pelvis and hip pain (100 patients). Nine studies set more than two controls, and six clinical trials lacked follow-up period.

3.2. Study Quality. The risk of bias of each included study as a checklist of quality is presented in Figure 2. Twenty-two trials specified the method of randomization, while one study stated that participants were randomized to groups but failed to provide a more detailed description [16], and one used a very simple allocation by randomly selected pieces of paper [17]. Twenty-one studies very well provided the experiences of acupuncturist, and three did not [18-20]. Nine studies do not mention the requirements of "deqi" sensation $[13,16-18$, 20-24]. Although it was not feasible to blind the participants when sham controlled efficacy trials were conducted, eight studies failed to provide the information about blinding [17, 19, 24-29] and two trials revealed that they did not perform blinding $[23,30]$. Eight trials did not report the blindness of outcome evaluators [16, 22-24, 27, 29, 31, 32], and one study revealed that they did not have blind evaluators [12]. Six trials did not conduct the follow-up research [12, 20, 26, 28, 29, 33]. Additionally, one of these trials failed to report baseline SPADI information [33]. 


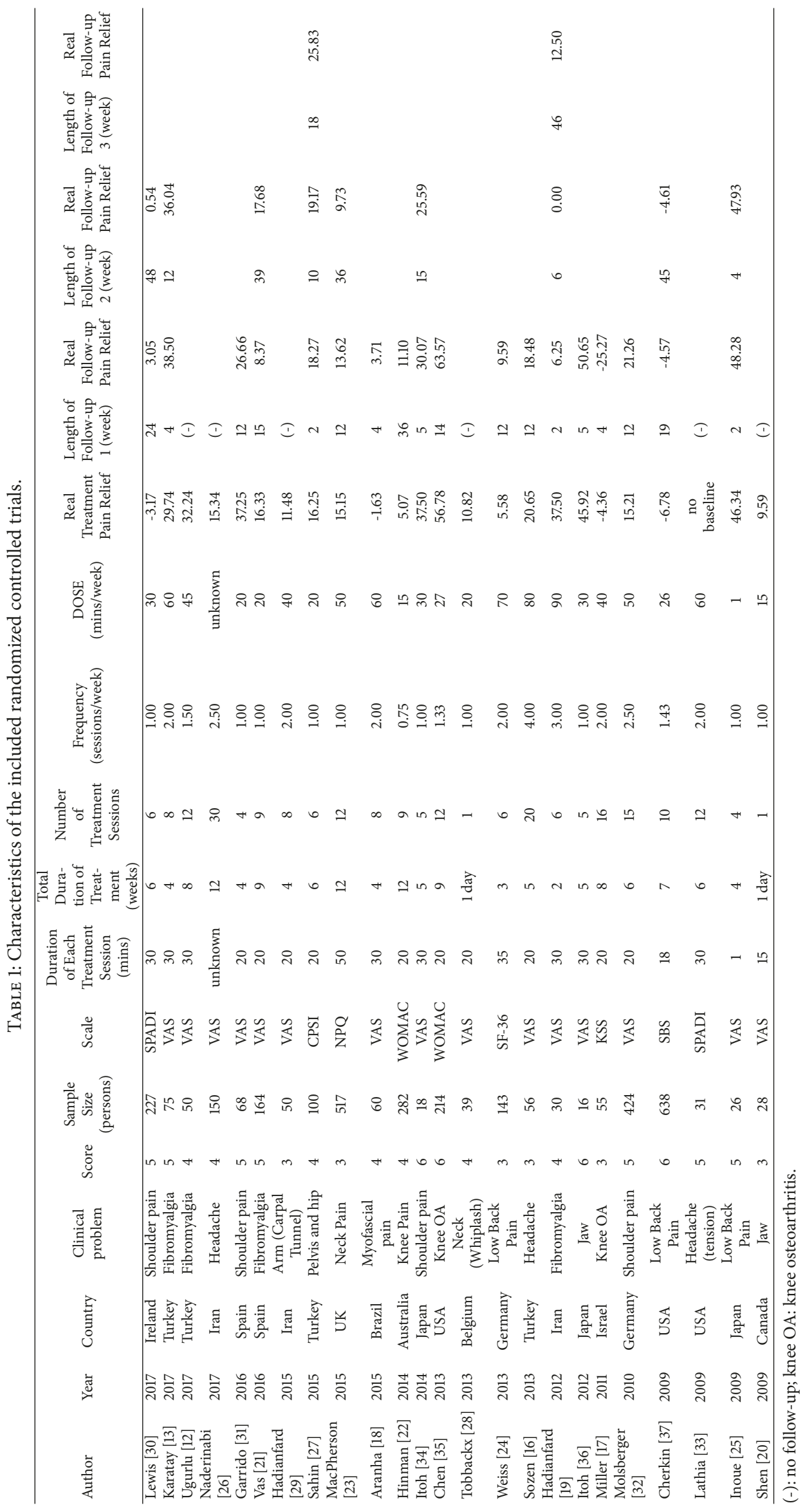


TABLE 2: Linear regression analysis on real treatment pain relief rate with schedule related factors.

\begin{tabular}{|c|c|c|c|c|}
\hline Dependent Variable & Explanatory Variable & Coefficient Estimates of Predictors & R-square Value & P-value \\
\hline \multirow{6}{*}{ Real Treatment Pain Relief Rate } & Duration of Each Treatment Session & -0.187 & 0.010 & 0.655 \\
\hline & Total Duration of Treatment & -0.429 & 0.007 & 0.710 \\
\hline & DOSE & -0.051 & 0.005 & 0.765 \\
\hline & Frequency & -1.343 & 0.004 & 0.771 \\
\hline & Number of Treatment Sessions & -0.310 & 0.014 & 0.592 \\
\hline & Score & 7.405 & 0.217 & 0.025 \\
\hline
\end{tabular}

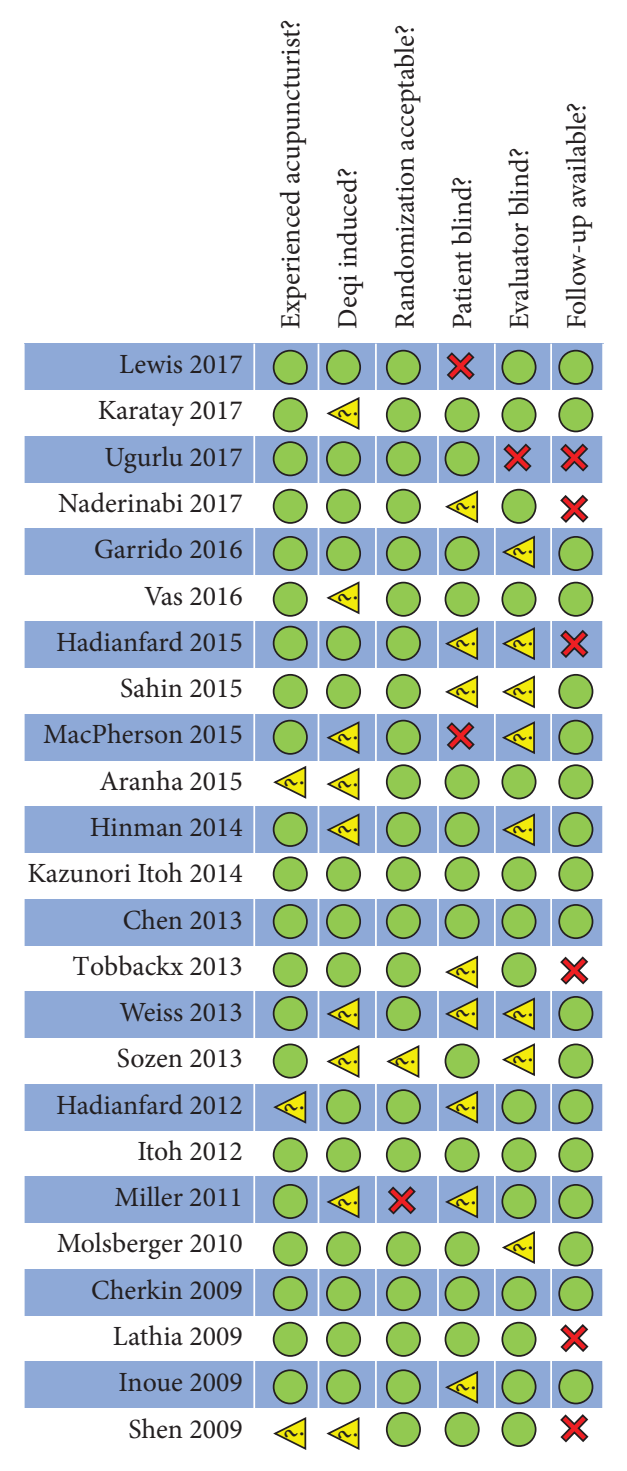

FIgURE 2: Risk of bias summary.

\subsection{Outcome Measurements}

3.3.1. Assessment of the Linear Regression Model between Real Treatment Pain Relief Rate and Schedule Related Factors. The correlation coefficients between each schedule related factor (sample size, duration of each treatment session, total duration of treatment, number of treatment sessions, frequency,
DOSE and score) and real treatment pain relief rate were analyzed as shown in Figure 3 and Table 2. Data showed that there was almost no liner relationship between each of other factors and real treatment pain relief rate, Figures 3(a)-3(e). In Figure 3(f), a positive correlation $(r=0.4654)$ was found between study quality score and pain relief rate after treatment. It indicated that study quality was associated with the size of acupuncture effect.

3.3.2. Assessment of the Linear Regression Model between Real Follow-up Pain Relief Rate and Schedule Related Factors. The results of the linear regression analysis were presented in Figure 4 and Table 3. From Figure 4(a), the result showed that the real follow-up pain relief rate declined with the extending of follow-up period.

In addition, the real follow-up pain relief rate was decreased with the increase of the duration of each treatment session and DOSE in Figures 4(b) and 4(f). Either shorting the treatment time of each session or decreasing the DOSE of treatment per week can achieve better treatment outcome. Meanwhile, it was noted that the real follow-up pain relief rate presented a positive correlation $(\mathrm{r}=0.3046)$ with study quality score (Figure $4(\mathrm{~g})$ ), which was the same with the real pain relief rate after treatment. The effect of the score on pain relief rate was maintained to the follow-up period.

3.3.3. Evaluation of the Schedule Related Factors That Affect Real Pain Relief Rate. In order to determine the appropriate frequency and DOSE of acupuncture treatment, each parameter was divided into several parts and one-way ANOVA analysis was carried out as shown in Figures 5(a) and 5(b). As we can see, lower frequency with 2 sessions/week and higher frequency greater than 2 sessions/week can achieve higher real treatment pain relief, while the one with 2 sessions/week can lead to lower pain relief, although there was no significant statistical difference among groups.

In Figure 5(b), the higher DOSE of acupuncture did not guarantee better treatment pain relief. The rate of pain relief increased slightly as the DOSE of acupuncture increased, peaked at the DOSE of $30 \mathrm{mins} /$ week, and then declined steadily along the extending of DOSE.

Furthermore, we separated follow-up weeks into 5 time points, and the pain relief effect of each time was analyzed in Figure 5(c). Up to the 18 weeks of follow-up, the rate of pain relief remained at a high level greater than $20 \%$ but it dropped sharply below $10 \%$ after 18 weeks. Thus, 18 weeks might be the expiry date of acupuncture treatment. 
TABLE 3: Linear regression analysis on real follow-up pain relief rate with schedule related factors.

\begin{tabular}{|c|c|c|c|c|}
\hline Dependent Variable & Explanatory Variable & Coefficient Estimates of Predictors & R-square Value & P-value \\
\hline \multirow{7}{*}{ Real Follow-up Pain Relief Rate } & Follow-up Weeks & -0.542 & 0.200 & 0.015 \\
\hline & Duration of Each Treatment Session & -0.562 & 0.117 & 0.070 \\
\hline & Total Duration of Treatment & -0.504 & 0.007 & 0.672 \\
\hline & Number of Treatment Sessions & -0.475 & 0.011 & 0.584 \\
\hline & Frequency & -4.008 & 0.037 & 0.320 \\
\hline & DOSE & -0.220 & 0.105 & 0.086 \\
\hline & Score & 5.219 & 0.093 & 0.108 \\
\hline
\end{tabular}

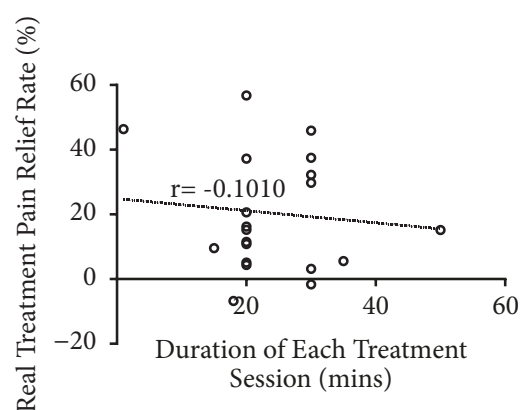

(a)

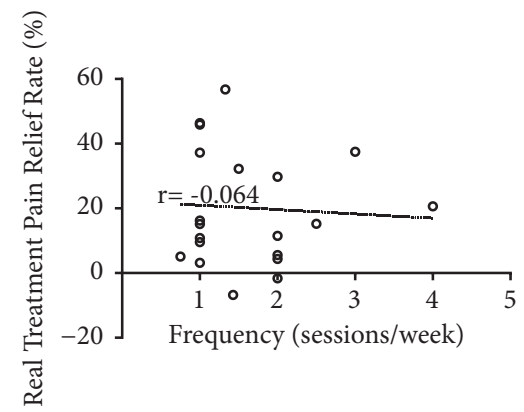

(d)

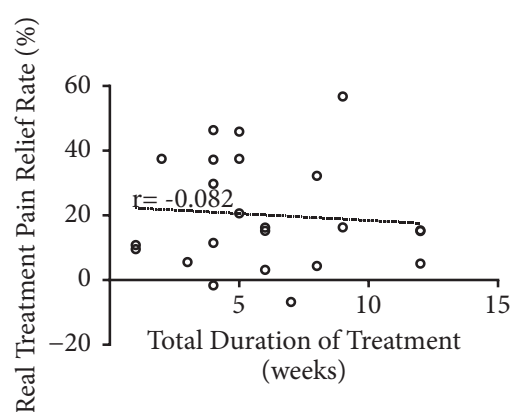

(b)

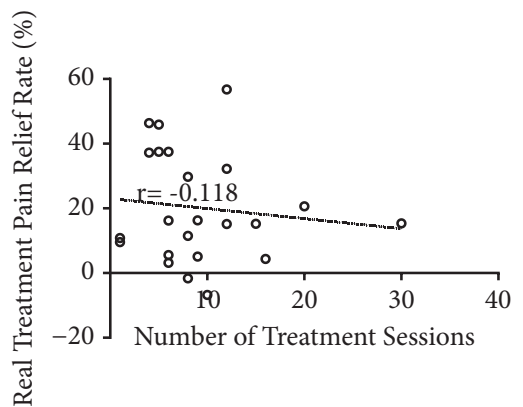

(e)

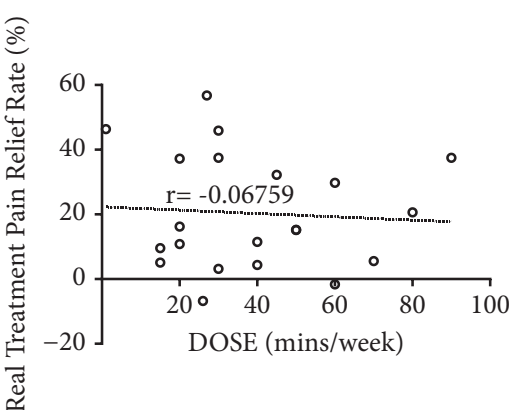

(c)

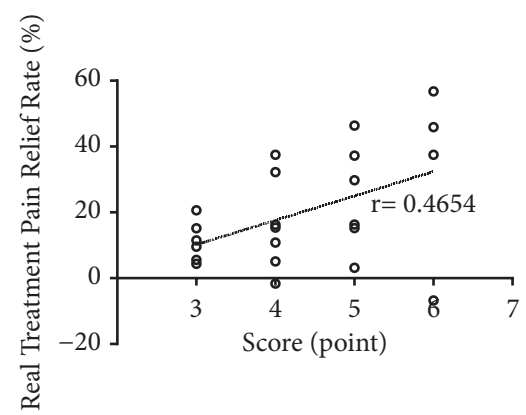

(f)

FIGURE 3: Correlation coefficients between real treatment pain relief rate and the following factors: (a) duration of each treatment session; (b) total duration of treatment; (c) DOSE; (d) frequency; (e) number of treatment sessions; (f) study quality score; the higher the score, the better the quality of the study.

Three-dimensional distribution diagram of three factors and pain relief rate in both treatment and follow-up period was shown in Figure 6. In Figure 6(a), most of those 22 trials showed effective acupuncture; the pain relief percentage in 9 trials was beyond 20\% (colored in green and blue), 15 trials above $10 \%$ (colored in yellow, green, and blue), 5 trials 1$10 \%$ (colored in orange), and 2 trials below $0 \%$ (colored in red). When the treatment schedule of two trials was the same, the points will be overlapped. From those 22 clinical trials, number of treatment sessions was set as 6-12 in 13 trials, total duration of treatment was costumed from 4 to 12 weeks in 18 trails, and 10 trials selected 20 mins and 7 trials chose 30 mins as the duration of each session.

In Figure 6(b), most of those 29 trials showed a long-term effective acupuncture; the pain relief percentage in 12 trials was beyond $20 \%$ (colored in bright yellow, green, and blue), 19 trials above $10 \%$ (colored in yellow, green, and blue), and 10 trials under $10 \%$ (colored in orange and red). When the treatment schedule of two trials is the same, the points will overlap.

\section{Discussion}

4.1. Strengths and Limitations. The review is a pilot to analyze randomized controlled trials of acupuncture treatment schedules for chronic pain; although Chinese trials and trials from Chinese databases are not assessed, specific acupuncture schedule that has been summarized with long-term clinical experience is usually used in the implementation of chronic pain randomized controlled trials conducted by 


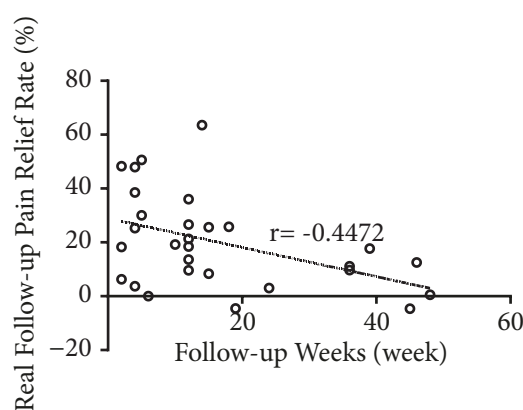

(a)

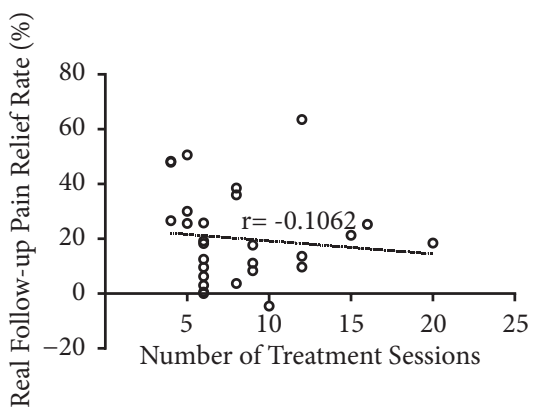

(d)

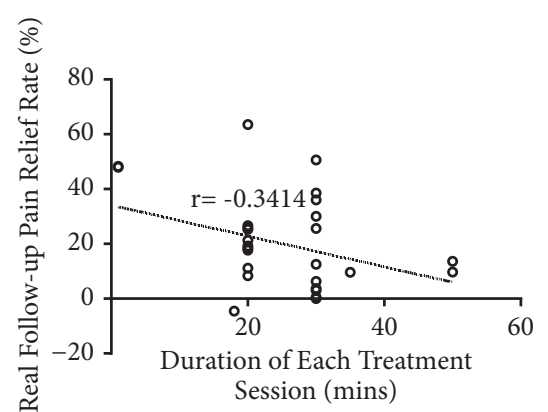

(b)

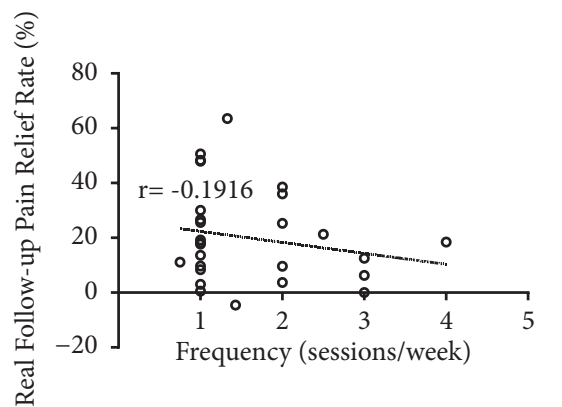

(e)

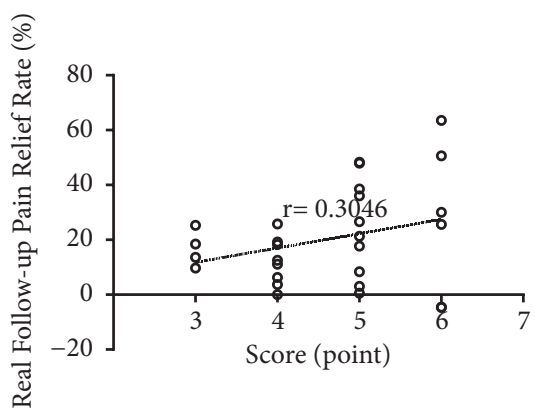

(g)

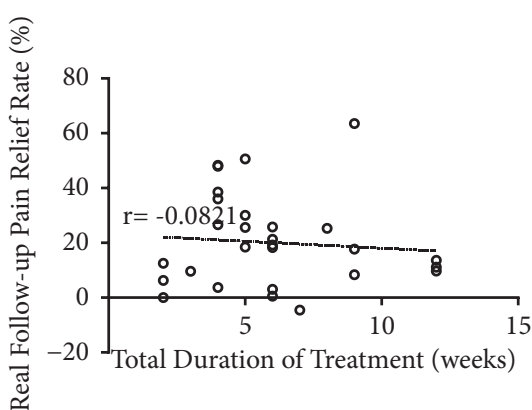

(c)

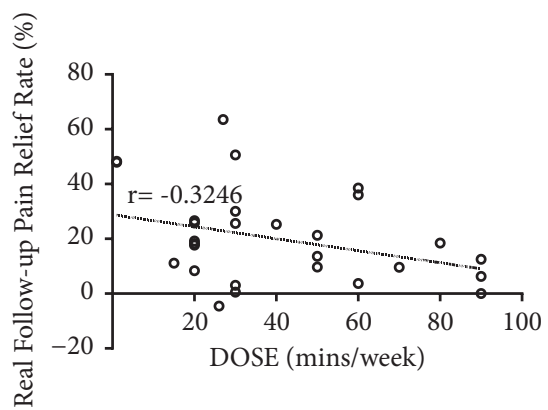

(f)

FIGURE 4: Correlation coefficients between real follow-up pain relief rate and the following factors: (a) follow-up weeks; (b) duration of each treatment session; (c) total duration of treatment; (d) DOSE; (e) frequency; (f) number of treatment sessions; (g) study quality score; the higher the score, the better the quality of the study.

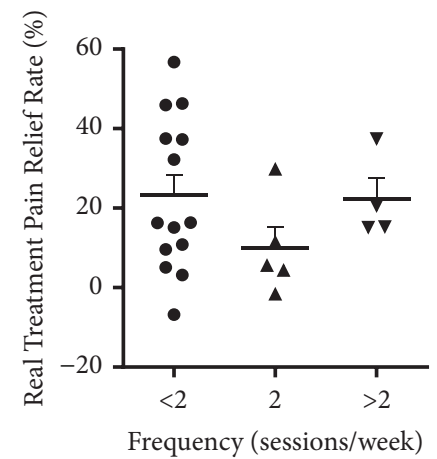

(a)

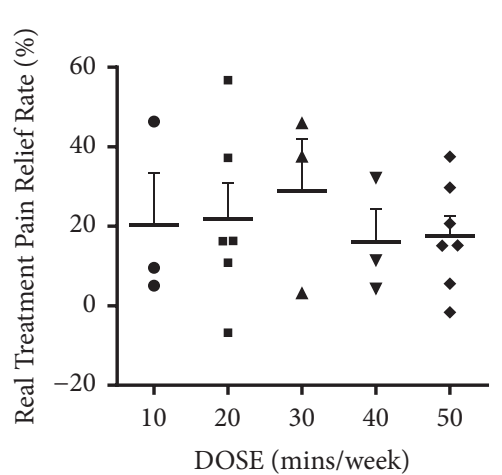

(b)

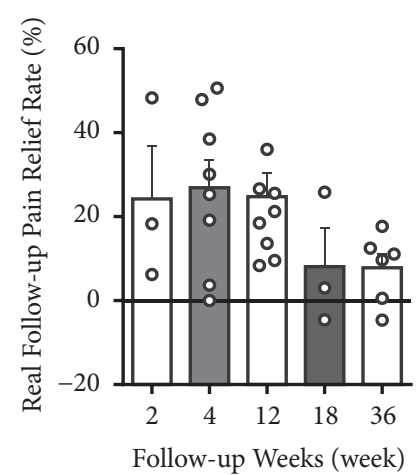

(c)

FIGURE 5: Relationship between acupuncture schedule related factors and pain relief rate. (a) The relationship between frequency and real treatment pain relief rate: frequency<2, including 3 sessions/4 weeks, 1 session/week, 4 sessions/ 3 weeks, 10 sessions/7 weeks, and 3 sessions/2 weeks; frequency=2, including 2 sessions/week; frequency $>2$, including 5 sessions/2 weeks, 3 sessions/week, and 4 sessions/week. (b) The relationship between DOSE and real treatment pain relief rate: DOSE was divided by cumulative stimulus time. (c) The relationship between follow-up weeks and real follow-up pain relief rate: follow-up weeks were divided by the length of weeks. 


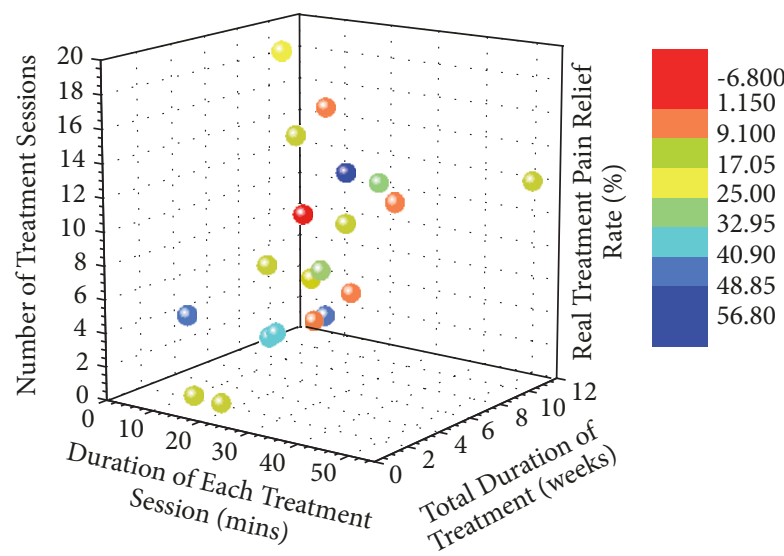

(a)

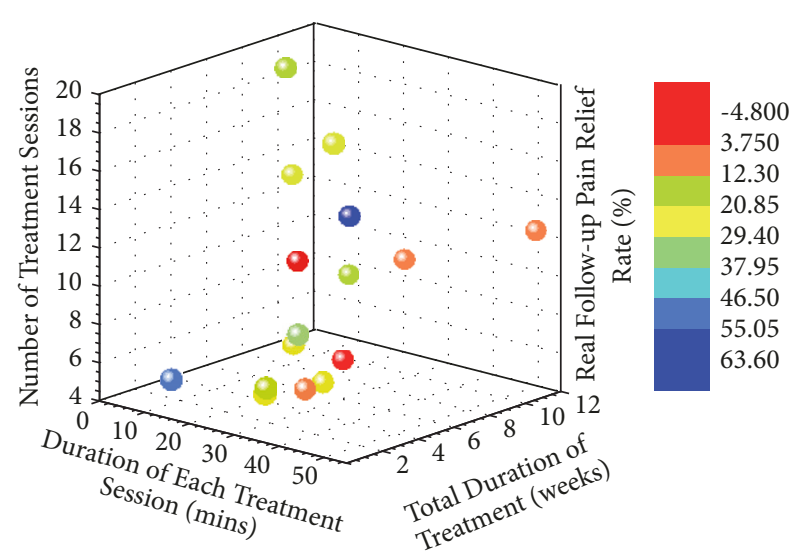

(b)

FIGURE 6: Three-dimensional distribution diagrams of three factors and pain relief rate. (a) Three factors (duration of each treatment session, total duration of treatment, and number of treatment sessions) and real treatment pain relief rate. (b) Three factors and real follow-up pain relief rate.

Chinese doctors. Randomized controlled trials set by westerners who are not aware of the importance of acupuncture schedule were reviewed in this study. Moreover, the included studies are confined to randomized controlled trials, which greatly reduced selection bias. Limitations of our review mainly focus on the strong heterogeneity of the studies and the limited number of trials, as well as the distribution of parameters. For example, length of each session is mostly conducted for 20-30 mins. What is more, we focused on treatment schedule without in-depth exploration of details, such as point selection, depth of insertion, stimulation method, and needle retention time. Furthermore, we studied the three main components, $\mathrm{D}, \mathrm{N}$, and T, of "DOSE", but other factors, e.g., deqi sensation, contributing to DOSE of acupuncture were not analyzed. However, linear regression coefficient is not a coefficient to measure causality. This data analysis should not be used directly as a basis for clinical treatment planning. Our primary aim is to draw attention to acupuncture schedules for protocol makers and detailed treatment protocols.

4.2. Interpretation of the Results. Firstly, the review implied that less frequency of acupuncture treatment can achieve the same effect just as that of higher frequency after treatment and less time in each session and less DOSE in weeks can achieve better follow-up outcome in patients with chronic pain. Our findings are highly consistent with some trials and analysis available in the literature. Zhang [38] reported that there was no difference between instant acupuncture and 30 min acupuncture in the contractile function of gallbladder. Shi [39] analyzed 350 cases of ophthalmic surgery performed with acupuncture anesthesia and found less time acupuncture was better than needle retention group. What is more, according to one of the earliest acupuncture books, Huang D $i$ Nei Jing Ling Shu [40], "the foot yang brilliance [conduit] is to be pierced 6 fen deep. [The needle] is to remain inserted for ten exhalations...The foot ceasing yin [qi conduits] is to be pierced 1 fen deep. [The needle] is to remain inserted for two exhalations." It means the needle remains inserted less than $1 \mathrm{~min}$. According to another classic book, Zhen Jiu Jia Yi Jing [41], among all the 154 acupoints, there are only 15 points needles inserted for more than 10 exhalations. The longest needle retention time was the points gongsun, neiting, and huantiao, all of which were for 20 exhalations, about $1 \mathrm{~min}$. Then in tang dynasty, Qian Jin Fang [42] wrote "a hundred exhalations between needles"; it means needle duration is about 5-6 mins. However, instant acupuncture was used by one of the included trials in the study. The number of instant acupuncture studies is too small to draw conclusions. If this result is confirmed, a lot of medical costs and social resources will be saved. Thus, further studies with more focus on duration of acupuncture session are suggested.

Secondly, our results show that the rate of pain relief dropped to the lower level after 18 weeks in the follow-up period. This suggests that the acupuncture treatment should be reconsidered at 18 weeks after needling to reinforce the effect. This new understanding could help to improve the long-term chronic pain control. The efficacy duration of acupuncture may be used as a criterion for efficacy evaluation in comparison with medicine and may provide new insight into mechanism research.

4.3. Implications for Future Research. We carefully evaluated the included trials and provided the following recommendations:

(1) Standard treatment protocols should be conducted in future randomized controlled trials to ensure the effectiveness of acupuncture on chronic pain.

(2) The recommendation treatment schedules should be added in STRICTA to evaluate the quality of performed randomized controlled trials.

(3) High-quality randomized controlled trials are warranted. Randomization, patients blinding, evaluator blinding, acupuncturist experience, and needling 
sensation should be clearly described in randomized controlled trials.

(4) Clinical effectiveness will be warranted if the time to receive acupuncture treatment and time when the retreatment is needed are clearly known.

(5) The schedule related factors of acupuncture, such as frequency, DOSE, and total duration of treatment, should be taken into account when designing clinical trials of acupuncture.

\section{Conclusions}

Lack of agreement on the appropriate schedule of acupuncture treatment is an obstacle the improvement of clinical effectiveness of acupuncture and research into acupuncture. An effective acupuncture therapy clearly involves more than schedule. According to the results various factors related to acupuncture schedule influence the effect of chronic pain controlling. Each factor might have an effect on pain relief in a certain range, which should be considered in designing and interpreting clinical trials. The most reliable method to determine the appropriate acupuncture schedule is to compare different schedule parameters in a tightly controlled condition. We recommend that more high-quality randomized controlled trials about treatment schedule research should be carried out and more details of the reference range of specific parameters should be provided.

\section{Conflicts of Interest}

The authors declare that there are no conflicts of interest.

\section{Authors' Contributions}

Yan-Jiao Chen searched the databases and wrote the manuscript. Yan-Jiao Chen and Cai-Tao Chen screened the articles, analyzed data, and created figures and tables. JiaYuan Liu checked all the data, and Gabriel Shimizu Bassi made corrections in the manuscript. Yong-Qing Yang provided financial support and reviewed the manuscript.

\section{Supplementary Materials}

Search Strategy in Pubmed, Embase, and Cochrane Central Register of Controlled Trials. (Supplementary Materials)

\section{References}

[1] I. Veith, "Acupuncture in traditional Chinese medicine. an historical review," California Medical, vol. 118, no. 2, pp. 70-79, 1973.

[2] "Use of acupuncture in modern health care," WHO Chronicle, vol. 34, no. 7-8, pp. 294-301, World Health Organization, Switzerland, 1980.

[3] Acupuncture: Review And Analysis of Reports on Controlled Clinical Trials, WHO, 2002.

[4] H. Wang, G. Yang, S. Wang, X. Zheng, W. Zhang, and Y. Li, “The most commonly treated acupuncture indications in the united states: a cross-sectional study," American Journal of Chinese Medicine, pp. 1-33, 2018.

[5] J. K. Phillips, M. A. Ford, R. J. Bonnie et al., Pain Management and the Opioid Epidemic: Balancing Societal and Individual Benefits and Risks of Prescription Opioid Use, National Academies Press, 2017.

[6] Z. Y. Ju, K. Wang, H. S. Cui et al., "Acupuncture for neuropathic pain in adults," Cochrane Database of Systematic Reviews, vol. 12, Article ID CD012057, 2057.

[7] E. Manheimer, K. Cheng, L. S. Wieland et al., "Acupuncture for hip osteoarthritis," Cochrane Database of Systematic Reviews, vol. 5, Article ID CD013010, 2018.

[8] H. Chen, Z. Ning, W. L. Lam et al., "Types of control in acupuncture clinical trials might affect the conclusion of the trials: a review of acupuncture on pain management," Journal of Acupuncture and Meridian Studies, vol. 9, no. 5, pp. 227-233, 2016.

[9] N. Takakura, M. Takayama, M. Nasu et al., "Patient blinding with blunt tip placebo acupuncture needles: comparison between $1 \mathrm{~mm}$ and $2 \mathrm{~mm}$ skin press," Journal of Integrative Medicine, vol. 16, no. 3, pp. 164-171, 2018.

[10] H. Lee, O. Kwon, J. Kim et al., "Efficacy and safety of acupuncture for functional constipation: a randomised, shamcontrolled pilot trial," BMC Complementary and Alternative Medicine, vol. 18, no. 1, aricle 186, 2018.

[11] I. Lund, J. Näslund, and T. Lundeberg, "Minimal acupuncture is not a valid placebo control in randomised controlled trials of acupuncture: a physiologist's perspective," Chinese Medicine, vol. 4 , no. 1, 2009.

[12] F. G. Ugurlu, N. Sezer, L. Aktekin et al., "The effects of acupuncture versus sham acupuncture in the treatment of fibromyalgia: a randomized controlled clinical trial," Acta Reumatológica Portuguesa, vol. 42, no. 1, pp. 32-37, 2017.

[13] S. Karatay, S. C. Okur, H. Uzkeser, K. Yildirim, and F. Akcay, "Effects of acupuncture treatment on fibromyalgia symptoms, serotonin, and substance P levels: a randomized sham and placebo-controlled clinical trial," Pain Medicine, vol. 19, no. 3, pp. 615-628, 2018.

[14] H. MacPherson, D. G. Altman, R. Hammerschlag et al., "Revised standards for reporting interventions in clinical trials of acupuncture (STRICTA): extending the CONSORT statement," PLoS Medicine, vol. 7, no. 6, Article ID e1000261, 2010.

[15] J. P. Higgins and S. Green, Cochrane Handbook for Systematic Reviews of Interventions, The Cochrane Collaboration, Oxford, UK, 2011.

[16] A. Sozen, M. Yilmaz, H. R. Koyuncuoglu, V. Yurekli, E. Uzar, and S. Kutluhan, "Effectiveness of acupuncture for the treatment of chronic daily headache: A sham-controlled clinical trial," Acta Medica Mediterranea, vol. 29, no. 2, pp. 167-172, 2013.

[17] E. Miller, Y. Maimon, Y. Rosenblatt et al., "Delayed effect of acupuncture treatment in OA of the knee: a blinded, randomized, controlled trial," Evidence-Based Complementary and Alternative Medicine, vol. 2011, Article ID 792975, 5 pages, 2011.

[18] M. F. Aranha, C. E. Müller, and M. B. Gavião, "Pain intensity and cervical range of motion in women with myofascial pain treated with acupuncture and electroacupuncture: a double-blinded, randomized clinical trial," Brazilian Journal of Physical Therapy, vol. 19, no. 1, pp. 34-43, 2014.

[19] M. J. Hadianfard and M. Hosseinzadeh Parizi, "A randomized clinical trial of fibromyalgia treatment with acupuncture compared with fluoxetine," Iranian Red Crescent Medical Journal, vol. 14, no. 10, pp. 631-640, 2012. 
[20] Y. F. Shen, J. Younger, G. Goddard, and S. Mackey, "Randomized clinical trial of acupuncture for myofascial pain of the jaw muscles.," Journal of Orofacial Pain, vol. 23, no. 4, pp. 353-359, 2009.

[21] J. Vas, K. Santos-Rey, R. Navarro-Pablo et al., "Acupuncture for fibromyalgia in primary care: a randomised controlled trial," Acupuncture in Medicine, vol. 34, no. 4, pp. 257-266, 2016.

[22] R. S. Hinman, P. McCrory, M. Pirotta et al., "Acupuncture for chronic knee pain a randomized clinical trial," Journal of the American Medical Association, vol. 312, no. 13, pp. 1313-1322, 2014.

[23] H. MacPherson, H. Tilbrook, S. Richmond et al., "Alexander technique lessons or acupuncture sessions for persons with chronic neck pain: a randomized trial," Annals of Internal Medicine, vol. 163, no. 9, pp. 653-662, 2015.

[24] J. Weiß, S. Quante, F. Xue, R. Muche, and M. Reuss-Borst, "Effectiveness and acceptance of acupuncture in patients with chronic low back pain: results of a prospective, randomized, controlled trial," The Journal of Alternative and Complementary Medicine, vol. 19, no. 12, pp. 935-941, 2013.

[25] M. Inoue, T. Hojo, M. Nakajima, H. Kitakoji, and M. Itoi, "Comparison of the effectiveness of acupuncture treatment and local anaesthetic injection for low back pain: A randomised controlled clinical trial," Acupuncture in Medicine, vol. 27, no. 4, pp. 174-177, 2009.

[26] B. Naderinabi, A. Saberi, M. Hashemi et al., "Acupuncture and botulinum toxin A injection in the treatment of chronic migraine: a randomized controlled study," Caspian Journal of Internal Medicine, vol. 8, no. 3, pp. 196-204, 2017.

[27] S. Sahin, M. Bicer, G. A. Eren et al., "Acupuncture relieves symptoms in chronic prostatitis/chronic pelvic pain syndrome: A randomized, sham-controlled trial," Prostate Cancer and Prostatic Diseases, vol. 18, no. 3, pp. 249-254, 2015.

[28] Y. Tobbackx, M. Meeus, L. Wauters et al., "Does acupuncture activate endogenous analgesia in chronic whiplash-associated disorders? a randomized crossover trial," European Journal of Pain, vol. 17, no. 2, pp. 279-289, 2013.

[29] M. Hadianfard, E. Bazrafshan, H. Momeninejad, and N. Jahani, "Efficacies of acupuncture and anti-inflammatory treatment for carpal tunnel syndrome," Journal of Acupuncture and Meridian Studies, vol. 8, no. 5, pp. 229-235, 2015.

[30] J. Lewis, J. Sim, and P. Barlas, "Acupuncture and electroacupuncture for people diagnosed with subacromial pain syndrome: a multicentre randomized trial," European Journal of Pain, vol. 21, no. 6, pp. 1007-1019, 2017.

[31] J. C. Rueda Garrido, J. Vas, and D. R. Lopez, "Acupuncture treatment of shoulder impingement syndrome: a randomized controlled trial," Complementary Therapies in Medicine, vol. 25, pp. 92-97, 2016.

[32] A. F. Molsberger, T. Schneider, H. Gotthardt, and A. Drabik, "German randomized acupuncture trial for chronic shoulder pain (GRASP) - a pragmatic, controlled, patient-blinded, multi-centre trial in an outpatient care environment," PAIN, vol. 151, no. 1, pp. 146-154, 2010.

[33] A. T. Lathia, S. M. Jung, and L. X. Chen, "Efficacy of acupuncture as a treatment for chronic shoulder pain," The Journal of Alternative and Complementary Medicine, vol. 15, no. 6, pp. 613618, 2009.

[34] K. Itoh, S. Saito, S. Sahara, Y. Naitoh, K. Imai, and H. Kitakoji, "Randomized trial of trigger point acupuncture treatment for chronic shoulder pain: a preliminary study," Journal of Acupuncture and Meridian Studies, vol. 7, no. 2, pp. 59-64, 2014.
[35] L. X. Chen, J. J. Mao, S. Fernandes et al., "Integrating acupuncture with exercise-based physical therapy for knee osteoarthritis: a randomized controlled trial," JCR: Journal of Clinical Rheumatology, vol. 19, no. 6, pp. 308-316, 2013.

[36] K. Itoh, S. Asai, H. Ohyabu, K. Imai, and H. Kitakoji, "Effects of trigger point acupuncture treatment on temporomandibular disorders: a preliminary randomized clinical trial," Journal of Acupuncture and Meridian Studies, vol. 5, no. 2, pp. 57-62, 2012.

[37] D. C. Cherkin, K. J. Sherman, A. L. Avins et al., "A randomized trial comparing acupuncture, simulated acupuncture, and usual care for chronic low back pain," JAMA Internal Medicine, vol. 169, no. 9, pp. 858-866, 2009.

[38] J. Q. Zhang and S. Z. Liu, "Observation on the effect of acupuncture on the contractile function of gallbladder," New Traditional Chinese Medicine, vol. 16, no. 1, pp. 29-30, 1986.

[39] L. S. Shi, "Clinical analysis of 350 cases of ophthalmic operation without retention under acupuncture and anesthesia," Tianjin Traditional Chinese Medicine, no. 6, pp. 32-33, 1988.

[40] P. U. Unschuld, Huang Di Nei Jing Ling Shu, University of California Oakland, Calif, USA, 2016.

[41] P. M. Huang, A-B Classic of Acupuncture and Moxibustion, Liaoning Science and Technology Press, Shenyang, China, 1997.

[42] S. M. Sun, Qian Jin Fang, Huaxia publishing house, Beijing, China, 1993. 


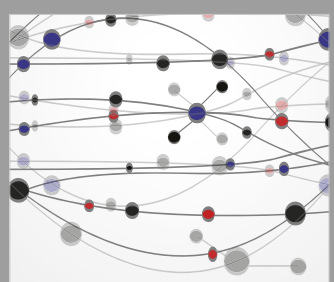

The Scientific World Journal
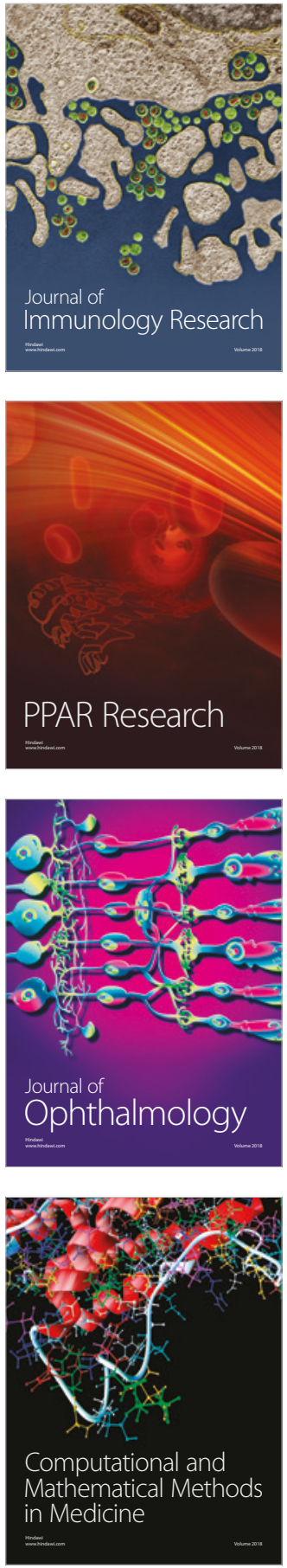

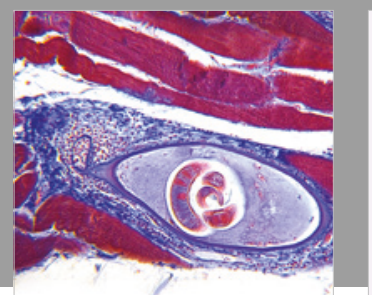

Gastroenterology Research and Practice

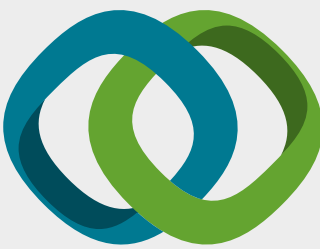

\section{Hindawi}

Submit your manuscripts at

www.hindawi.com
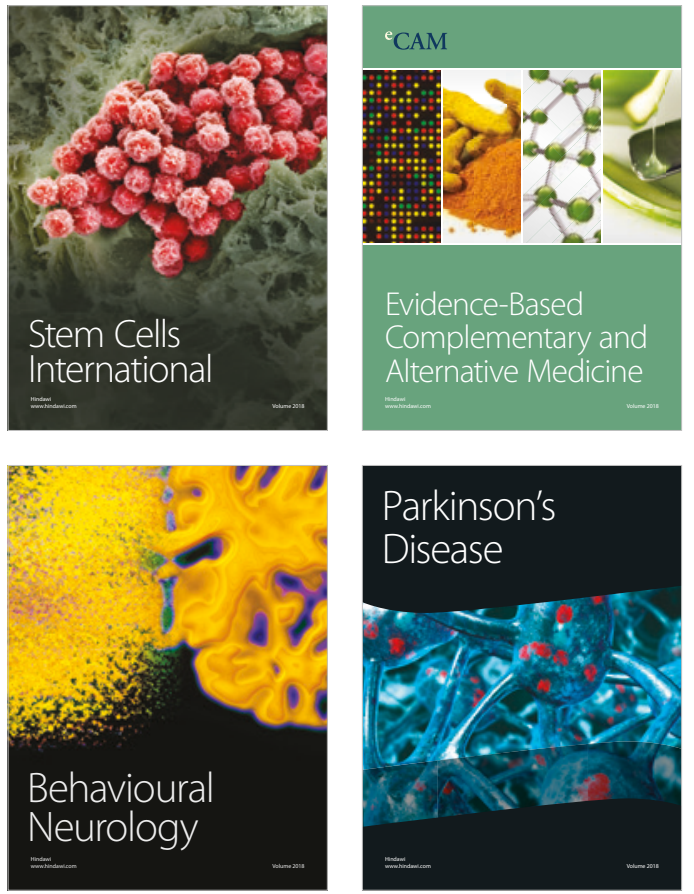

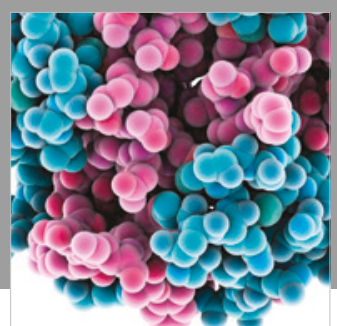

ournal of

Diabetes Research

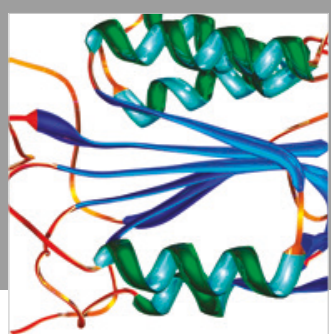

Disease Markers
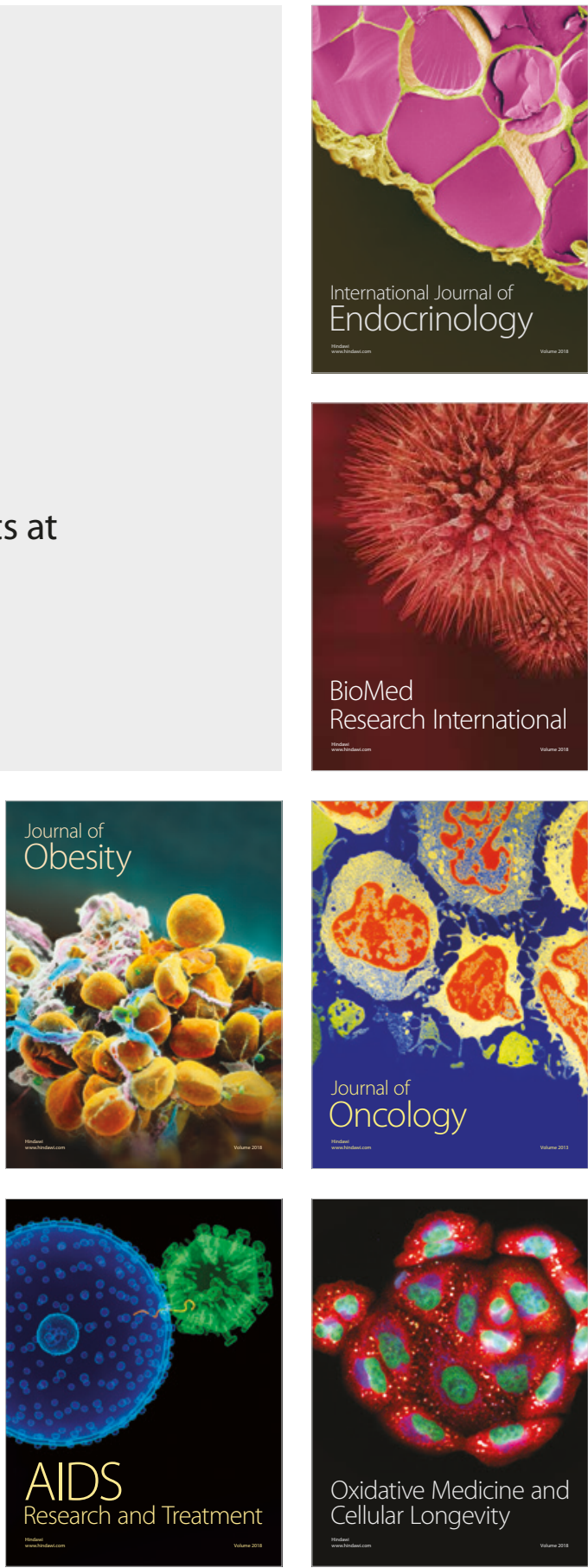